\title{
Nonlinear Bingham Model of a Magnetorheological Shock Absorber
}

\author{
Yonggang YANG, Peng YU \& Shixing ZHU \\ College of Aeronautical Engineering, Civil Aviation University of China, China
}

\begin{abstract}
A structure of magnetorheological shock absorber is introduced to overcome the defect of passive control of oleo-pneumatic shock absorbers. Its damper characteristics are test by vibration test bench, and nonlinear Bingham model is used to characterize the intrinsic nonlinear behavior of shock absorber. The parameters of damper model are identified by least square method according the experiment data. Compared with the results of experiment, the nonlinear Bingham model can effectively characterize the behavior of shock absorber.
\end{abstract}

KEYWORD: Magnetorheological shock absorber; Nonlinear Bingham mode; Least square method.

\section{INTRODUCTION}

Aircraft landing gear shock absorber, as an important component of the aircraft, plays an irreplaceable role in bearing the static and dynamic load when plane contacted with the ground, absorbing and dissipating the energy produced in the landing impact and the runway. Currently, many applications in the landing gear use passive control of oleo-pneumatic shock absorbers, these shock absorbers with a variable cross-section metering pin or double chambers or other structures to improve damping properties of shock absorbers [1-3]. In most cases the proper performance of typically used shock absorbers is achieved by introducing orifices equipped with metering pins, which are able to control the internal fluid flow depending on the deflection of the piston. landing gear absorbers Passive controlled has the characteristics of simple structure, light weight, easy repair, high security. But the performance is always optimized for a particular type of touching down without taking into account variation of the landing conditions. The classically made struts are expected to be designed to perform in everyday exploitation, as well as in emergency situations, which is objectively unachievable [4]. An other type absorber is considered [5-10], which can change the damping force in a range accoding to the vibration. YoungTai Choi and Norman M. Wereley demonstrated the feasibility and effectiveness of electrorheological (ER) and magnetorheological (MR) fluid-based landing gear systems on attenuating dynamic load and vibration due to the landing impact [11], Disha
Saxena et al utilized fuzzy Control for vibration control systems of the developed MR damper for landing gear system, to attenuate the sustained vibrations during the landing phase [12]. Over the last decade, MR damping technology has been rapid developed, and MR damping fluid medium for the MR shock absorber have been gradually applied in automobiles, bridges, buildings, etc. [13-14]. Since the MR damper has a simple structure, low energy consumption, rapid response and damping adjustment range, etc., to be used in aircraft landing gear shock absorber damping system will greatly improve the performance of the landing gear. In this paper, a new structure of shock absorber based on MR dampers is introduced and its damping force model is built.

\section{STRUCTURE OF MR SHOCK ABSORBER}

To ensure reliability and shock absorber damping force adjustable damping properties, based on conventional oleo-pneumatic shock absorber with metering pin, a shock absorber based on MR damper with metering pin, which is also called MR shock absorber, is designed, so that it's damper effect can be simultaneously controlled by current and stroke, then which will improve efficiency and damping force adjustment range while improve the safety factor of the shock absorber. Its structure diagram is shown in Fig.1.

In operating the MR shock absorber, piston rod drives the guide plate to press against the chamber, 
the MR fluid on the upper end of guide plate flows through the orifice on the top of piston rod, then through the upper portion hollow of the piston rod, finally through annular gap of piston by flowing through the radial orifice in the piston rod. The piston of the shock absorber is axially provided with a number of rectangular annular grooves. On the action of external electromagnetic field, the MR fluid flows through the rectangular grooves with the expansion of its volume and decrease of its velocity. Because of the dissipation effect of the rectangular grooves produced vortex, the kinetic energy is turned into heat energy, so MR shock absorber with rectangular annular groove has the effect of enhancing the damping force. In addition, by using tapered metering pin, damping force of the shock absorber varies with the stroke change, and when the semi-active control of MR damper is failure, the shock absorber can degenerate into an efficient oleopneumatic shock absorber, which makes landing gear shock absorber system more secure.

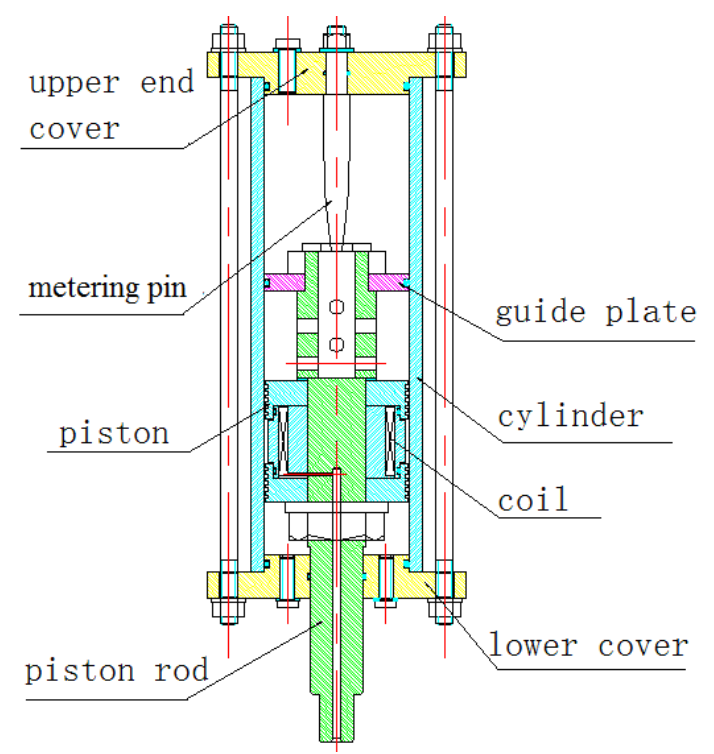

Fig.1 The structure diagram of shock absorber based on MR damper with metering pin

\section{NONLINEAR BINGHAM DAMPER MODEL}

Effective control of an MR damper mainly depends on understanding its nonlinear hysteretic behavior under an applied magnetic field. Therefore, one needs to develop control algorithms that take maximum advantage of the unique features of MR dampers, and the models must adequately characterize the intrinsic nonlinear behavior of the devices. A lot of models and their improved models have been proposed to characterize the intrinsic nonlinear behavior of these devices, such as Bingham model [15], Bouc-wen model[16], Dahl hysteresis model [17] neural networks model [18], and so on. In this paper, an improved bingham model is chosen to represent the MR fluid behavior.
Bingham viscoplastic model is developed by Stanway et al. It can be expressed as

$$
\mathrm{F}=f_{\mathrm{c}} \operatorname{sgn}(\mathrm{v})+c_{0} \mathrm{v}+f_{0}
$$

Where viscous damping coefficient $c_{0}$, Coulomb friciton $f_{\mathrm{c}}$ and compensatory force $f_{0}$, existing with compensator. In this model, a Coulomb friction element is placed in parallel with a linear viscous damping, which schematized in Fig. 2.
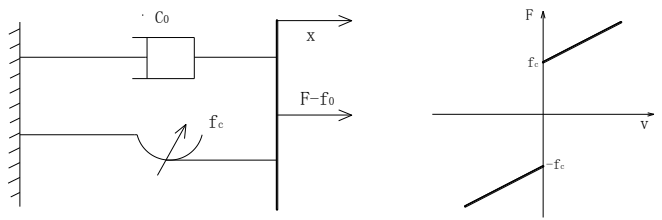

Fig.2 Bingham Model

Bingham model can well match the relation of damping force-displacement of MR damper, but not well fit the relation of force - velocity, especially where velocity is very small and the displacement and velocity with the same direction. In fact, the damping force of MR damper have obvious nonlinear behavior when its speed is slow, where Bingham model describe the behavior by linear viscous damping, it is a difference with the actual. Q. Zhou et al proposed an improved nonlinear Bingham model based on Bingham model [19]. Z. L. Liu makes a further improvement by using nonlinear damping element expressed by polynomial to replace the linear viscous damping in Bingham model [20]. The model can be expressed as

$$
\mathrm{F}=f_{\mathrm{c}} \operatorname{sign}(\mathrm{v})+\left(c_{\mathrm{o}}+c_{1}|v|+c_{2}|v|^{2}+\mathrm{c}_{3}|v|^{3}\right) \mathrm{v}+f_{0}
$$

Where damping coefficient constant term of nonlinear viscous damper $c_{0}$, damping coefficient one degree term of nonlinear viscous damper $c_{1}$, damping coefficient quadratic term of nonlinear viscous damper $c_{2}$, and damping coefficient cubic term of nonlinear viscous damper $c_{3}$.

Due to the presence of high order term of velocity, the model may reflect the force-velocity relation in case of high frequency situation, more suitable for MR absorber of the actual working conditions in landing gear, so this model is used. All damping coefficient terms are expressed by cubic polynomial expression of current according to characteristic test of MR fluid done by manufacturer to replace the quadratic polynomial expression in [20]. The relations of coefficient and current are expressed as

$$
\begin{aligned}
& f_{c}=f_{c 1} i^{3}+f_{c 2} i^{2}+f_{c 3} i+f_{c 0} \\
& c_{0}=c_{01} i^{3}+c_{02} i^{2}+c_{03} i+c_{00} \\
& c_{1}=c_{11} i^{3}+c_{12} i^{2}+c_{13} i+c_{10} \\
& c_{2}=c_{21} i^{3}+c_{22} i^{2}+c_{23} i+c_{20}
\end{aligned}
$$


4 CHARACTERISTIC TEST AND DAMPER MODEL CONSTRUCTION OF THE SHOCK ABSORBER

\subsection{Characteristic test of the shock absorber}

In order to get the shock absorber damper characteristic, vibration test bench which has one degree of freedom to provide vertical reciprocating vibration is utilized. Reciprocating move is imposed to the shock absorber, and the values of force, velocity can be got from the sensors. At every test, the value of current remains const to ensure the magnetic field applied to the magnetor-heological damper unchanged. In the combination of different amplitudes, different frequencies and different current, shock absorber damper is obtained. Fig.3 shows the curves of force-velocity when amplitude is $3 \mathrm{~mm}$, and frequency is $4 \mathrm{HZ}$, and the current is respectively $0 \mathrm{~A}, 0.1 \mathrm{~A}, 0.3 \mathrm{~A}, 0.5 \mathrm{~A}$. Fig.4 shows the curves of force-displacement on the same conditions.

\subsection{Damper model construction of the shock absorber}

Parameters in formula (3) are identified by least square method according to the experiment result of characteristic test. There values are shown in table 1 . The coefficients in formula (2) can be expressed as

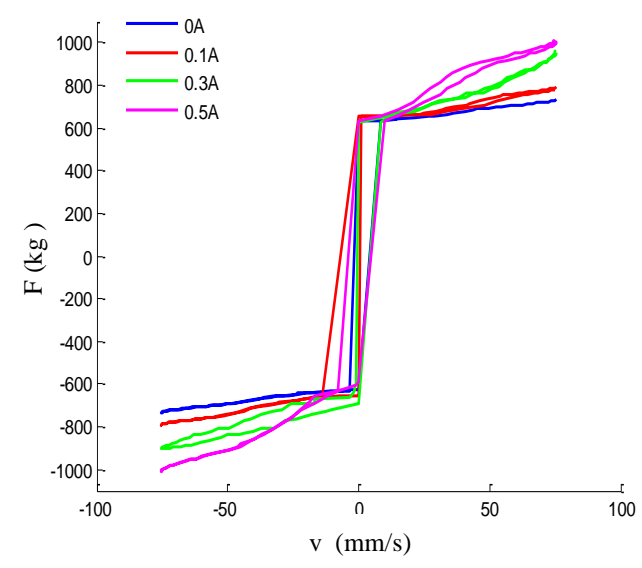

Fig.3 Curves of force-velocity

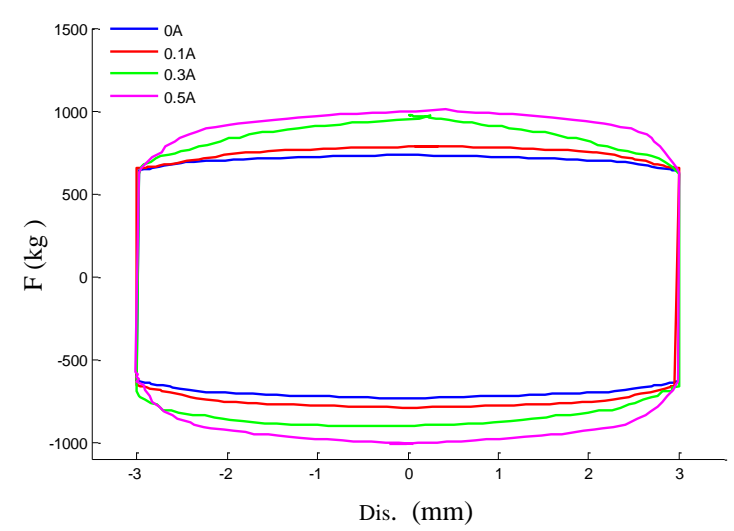

Fig.4 Curves of force- displacement

Table 1 Identified parameters' value in Bingham model

\begin{tabular}{|c|c|c|c|c|}
\hline parameters & $f_{\mathrm{c} 0}(\mathrm{~kg})$ & $f_{\mathrm{c} 1}\left(\mathrm{~kg}^{*} \mathrm{~A}^{-1}\right)$ & $f_{\mathrm{c} 2}\left(\mathrm{~kg}^{*} \mathrm{~A}^{-2}\right)$ & $f_{\mathrm{c} 3}\left(\mathrm{~kg} * \mathrm{~A}^{-3}\right)$ \\
\hline value & 588.1 & 139.2 & -282.1 & 251 \\
\hline parameters & $c_{01}\left(\mathrm{~kg}^{*} \mathrm{~mm}^{-1 *} \mathrm{~s}\right)$ & $c_{02}\left(\mathrm{~kg}^{*} \mathrm{~mm}^{-1 *} \mathrm{~s}^{*} \mathrm{~A}^{-1}\right)$ & $c_{03}\left(\mathrm{~kg}^{*} \mathrm{~mm}^{-1 *} \mathrm{~s}^{*} \mathrm{~A}^{-2}\right)$ & $c_{00}\left(\mathrm{~kg}^{*} \mathrm{~mm}^{-1 *} \mathrm{~s}^{*} \mathrm{~A}^{-3}\right)$ \\
\hline value & 0.5259 & 6.404 & 14.82 & -0.004167 \\
\hline parameters & $c_{11}\left(\mathrm{~kg}^{*} \mathrm{~mm}^{-1 *} \mathrm{~s}\right)$ & $c_{12}\left(\mathrm{~kg}^{*} \mathrm{~mm}^{-1 *} \mathrm{~s}^{*} \mathrm{~A}^{-1}\right)$ & $c_{13}\left(\mathrm{~kg}^{*} \mathrm{~mm}^{-1 *} \mathrm{~s}^{*} \mathrm{~A}^{-2}\right)$ & $c_{10}\left(\mathrm{~kg}^{*} \mathrm{~mm}^{-1 *} \mathrm{~s}^{*} \mathrm{~A}^{-3}\right)$ \\
\hline value & -0.08128 & -0.1248 & 1.922 & -2.762 \\
\hline parameters & $c_{21}\left(\mathrm{~kg}^{*} \mathrm{~mm}^{-1 *} \mathrm{~s}\right)$ & $c_{22}\left(\mathrm{~kg}^{*} \mathrm{~mm}^{-1 *} \mathrm{~s}^{*} \mathrm{~A}^{-1}\right)$ & $c_{23}\left(\mathrm{~kg}^{*} \mathrm{~mm}^{-1 *} \mathrm{~s}^{*} \mathrm{~A}^{-2}\right)$ & $c_{20}\left(\mathrm{~kg}^{*} \mathrm{~mm}^{-1 *} \mathrm{~s}^{*} \mathrm{~A}^{-3}\right)$ \\
\hline value & -0.0008527 & 0.03102 & -0.003369 & -0.0007472 \\
\hline
\end{tabular}

$$
\begin{aligned}
f_{c}= & 251 i^{3}-282.1 i^{2}+139.2 i+588.1 \\
c_{0}= & -0.00416 i^{3}+14.82 i^{2}+6.404 i+0.5259 \\
c_{1}= & -2.762 i^{3}+1.922 i^{2}-0.1248 i-0.08182 \\
c_{2}= & -7.472 \times 10^{-4} i^{3}-3.369 \times 10^{-3} i^{2} \\
& +3.102 \times 10^{-2} i-8.5267 \times 10^{-4}
\end{aligned}
$$

In order to verify the generality of nonlinear Bingham model, some other experiments are done, and the results respectively gotten from experiments, Bingham model and nonlinear Bingham model are compared, which are shown in Fig. 5.

As Fig.5 shown, the fitting accuracy of nonlinear Bingham model is significantly higher than that of Bingham model, and the output force relative error of nonlinear Bingham model value is much smaller than that of Bingham model. The nonlinear Bingham model can effectively represent the relation of forcedisplacement of the shock absorber.

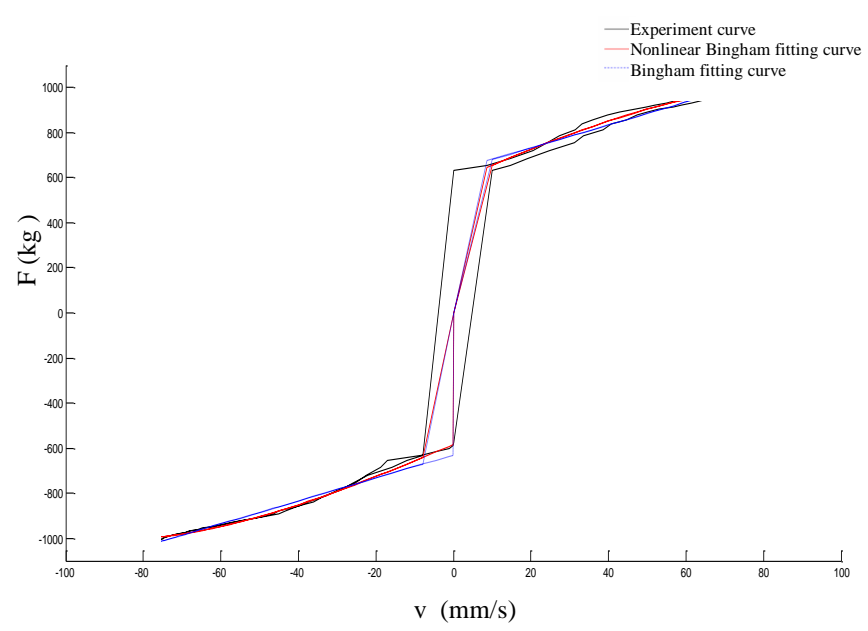

(a) The curve of force-velocity 


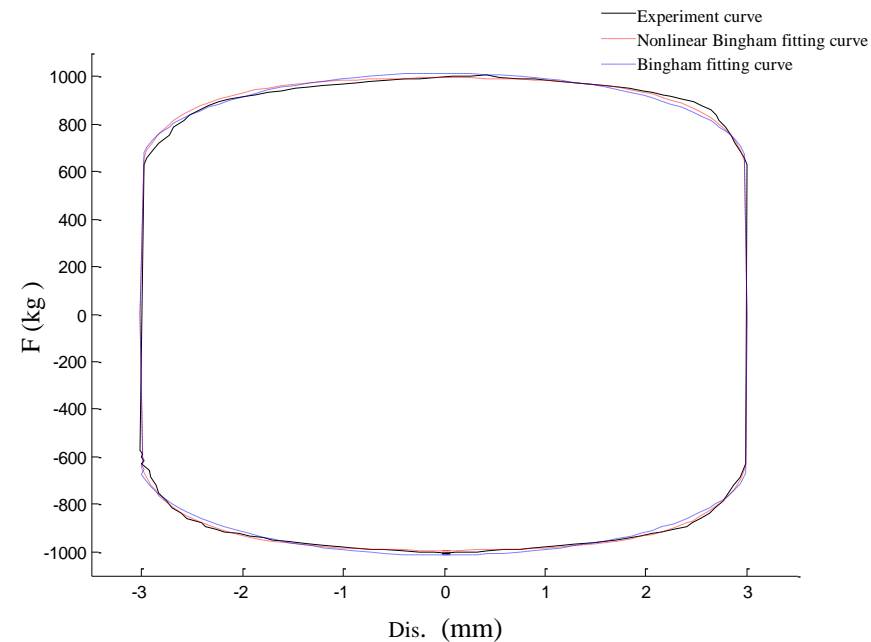

(b) The curve of force-displacement

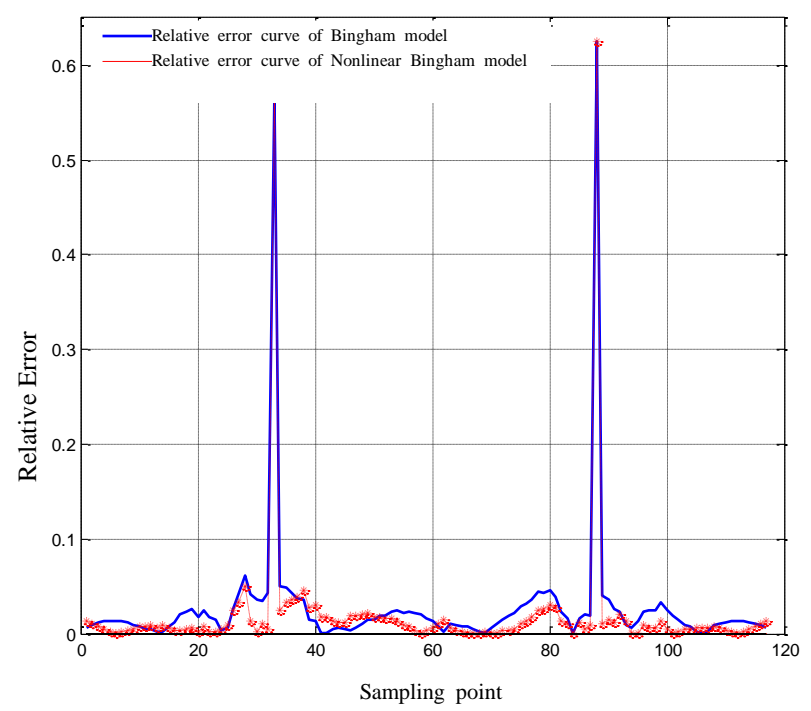

(c) The curve of error

Fig.5 Comparison of experiment result and model result

\section{CONCLUSION}

In this paper, a new structure of shock absorber based on magnetorheological is introduced to replace the oleo-pneumatic shock absorbers, and the damper characteristics of magnetorheological shock absorber are test by vibration test bench by change the amplitude, frequency and current. Bingham model and Nonlinear Bingham model are used to characterize the intrinsic nonlinear behavior of shock absorber. And the effectiveness of the damper model is respectively compared with the results of vibration test experiments and Bingham Model.

\section{ACKNOWLEDGMENTS}

The authors would like to thank the anonymous referees for their valuable suggestions. The research work was supported by National Natural Science Foundation of China under Grant No. 61172013 and the Fundamental Research Funds for the Central Universities under Grant No. 3122013C010.

\section{REFERENCES}

[1] Liu Hui, Gu Hongbin, Chen Dawei. 2008, Application of High-speed Solenoid Valve to the Semi-active Control of Landing Gear, Chinese Journal of Aeronautics

[2] Aircraft design manual editorial committee. 2002. Aircraft design manual, 14th books: take-off landing system design. Beijing: aviation industry press.

[3] Zhu Shuhua, Tong Mingbo, Xu Jie. 2009. Design of sign of new passive adaptive shock absorber of landing gear and study of its landing performance. Transactions of Nanjing University of Aeronautics \&Astronautics.

[4] G. Mikulowski, J. Holnichi-szulc. 2003. Adaptive aircraft shock absorbers. AMAS Workshop on Smart Materials and Structures, Jadwisin.

[5] Batterbee D. C., Sims N. D., Stanway R.. 2007. Magnetorheological Landing Gear: 1. A Design Methodology. Smart Mate rials and Structures.

[6] Batterbee D. C., Sims N. D., Stanway R.. 2007. Magnetorheological Landing Gear: 2. Validation Using Experimental Data. Smart Materials and Structures.

[7] Jia Yuhong, Wu Xiaojuan. 2007. Fuzzy control of landing gear based on M R damper. Journal of Beijing University of Aeronautics and Astronautics.

[8] LIU Hui, GU Hongbin, WU Dongsu. 2006. Shock absorber performance study of semi-active control of landing gear.Acta aeronautica et astronautica sinica.

[9] Wang Wei, Xia Pinqi. 2007. Adaptive Control of Helicopter Ground Resonance with Magnetorheological Damper. Chinese Journal of Aeronautics.

[10] TIAN Jing, DING Li, KONG Ling-shuai, ZHU Shi-xing. 2012. Simulation and Analysis of Semi-Active Control for MR Damper Landing Gear. Chinese Hydraulics \& Pneumatics.

[11] Choi Y T, Wereley N M. 2003. Vibration Control of a Landing Gear System Featuring Electrorheological /Magnetorheological Fluids. Journal of Aircraft.

[12] Disha Saxena, Harsh Rathore. 2013. Vibration Control of MR Damper Landing Gear. International Journal of Advanced Research in Artificial Intelligence.

[13] He jie, Gao lixia, Long zheng, Liu yongzhi, Liu xiumei. 2013. Theoretic and experimental study of chainformation mechanism for MRF. Jounal of functional materials, January.

[14] Zhou yun, Tan ping. 2007. Magnetorheological damping control theory and technology, Beijing:Science press.

[15] Stanway R, Sproston. 1987. Nonlinear modeling of an electrorheological vibration damper. Electrostatics.

[16] Nitin Ambhore, Shyamsundar Hivarale, Dr. D. R. Pangavhane. 2013. A Study of Bouc-Wen Model of Magnetorheological Fluid for Damper Vibration Control. International Journal of Engineering Research \& Technology.

[17] Dahl P. R. 1976. Solid friction damping of mechanical vibrations, AIAA.

[18] Wang D H and Liao W H. 2005. Modeling and control of magnetorheological fluid dampers using neural networks. Smart Material. Structure.

[19] Qiang, Zhou Wei-lian Ju. 2002. Two kinds of force models of Magnetorheological damper and test Validation. Journal of Earthquake Engineering and Seismic Vibration.

[20] Zhong-liang Liu. 2012. The establishment of the dynamics model of Magnetorheological Damper and its numerical simulation research. Changsha. Central South University. 